\title{
Nailfold Capillaroscopy Characteristics of Antisynthetase Syndrome and Possible Clinical Associations: Results of a Multicenter International Study
}

\author{
Marco Sebastiani, Konstantinos Triantafyllias, Andreina Manfredi, Miguel Angel González-Gay, \\ Natalia Palmou-Fontana, Giulia Cassone, Ulrich Drott, Christiane Delbrück, \\ Jorge Rojas-Serrano, Chiara Bertolazzi, Laura Nuño, Margherita Giannini, Florenzo Iannone, \\ Esther F. Vicente, Santos Castañeda, Albert Selva-O’Callaghan, Ernesto Trallero Araguas, \\ Giacomo Emmi, Annamaria Iuliano, Jutta Bauhammer, Nikolaus Miehle, Simone Parisi, \\ Lorenzo Cavagna, Veronica Codullo, Carlomaurizio Montecucco, Francisco Javier Lopez-Longo, \\ Julia Martínez-Barrio, Juan Carlos Nieto-González, Silvia Vichi, Marco Confalonieri, \\ Paola Tomietto, Raoul Bergner, Alberto Sulli, Francesco Bonella, Federica Furini, \\ Carlo Alberto Scirè, Alessandra Bortoluzzi, Christof Specker, Simone Barsotti, Rossella Neri, \\ Marta Mosca, Marzia Caproni, Julia Weinmann-Menke, Andreas Schwarting, Vanessa Smith \\ and Maurizio Cutolo, and the American and European Network of Antisynthetase Syndrome \\ Collaborative Group
}

\begin{abstract}
Objective. To describe nailfold videocapillaroscopy (NVC) features of patients with antisynthetase syndrome (AS) and to investigate possible correlations with clinical and serological features of the disease.

Methods. We retrospectively analyzed NVC images of 190 patients with AS [females/males 3.63, mean age $49.7 \pm 12.8$ yrs, median disease duration 53.7 mos (interquartile range 82), 133 anti-Jo1 and 57 non-anti-Jo1-positive patients]. For each patient, we examined number of capillaries, giant capillaries, microhemorrhages, avascular areas, ramified capillaries, and the presence of systemic sclerosis (SSc)-like pattern. Finally, we correlated NVC features with clinical and serological findings of patients with AS. Concomitantly, a historical cohort of 75 patients with antinuclear antibody-negative primary Raynaud phenomenon (RP) and longterm followup was used as a control group (female/male ratio 4.13/1, mean age $53.9 \pm 17.6 \mathrm{yrs}$ ) for NVC measures.

Results. NVC abnormalities were observed in $62.1 \%$ of AS patients compared with $29.3 \%$ of primary RP group ( $p<0.001)$. An SSc-like pattern was detected in 67 patients $(35.3 \%)$ and it was associated with anti-Jo1 antibodies $(p=0.002)$ and also with a longer disease duration $(p=0.004)$. Interestingly, there was no significant correlation between the presence of SSc-like pattern and RP, and only $47 \%$ of patients with SSc-like pattern had RP.

Conclusion. NVC abnormalities are commonly observed in AS, independently from the occurrence of RP. The presence of an SSc-like pattern could allow identification of a more defined AS subtype, and prospective studies could confirm the association with clinical and serological features of AS. (First Release November 15 2018; J Rheumatol 2019;46:279-84; doi:10.3899/jrheum.180355)
\end{abstract}

Key Indexing Terms:

ANTISYNTHETASE SYNDROME

RAYNAUD PHENOMENON

ANTISYNTHETASE ANTIBODIES

SYSTEMIC SCLEROSIS PATTERN

NAILFOLD VIDEOCAPILLAROSCOPY

From the Rheumatology Unit, Azienda Policlinico of Modena, University of Modena and Reggio Emilia, Modena, Italy; ACURA Rheumatology Center, Bad Kreuznach, Germany; Rheumatology Division, Hospital Universitario Marqués de Valdecilla, IDiVAL, University of Cantabria, Santander, Spain; Rheumatology Division, University Hospital of Frankfurt, Frankfurt, Germany; Interstitial Lung Disease and Rheumatology Unit, Instituto Nacional de Enfermedades Respiratorias,
Ismael Cosío Villegas, Mexico City, Mexico; Servicio de Reumatología, Hospital Universitario La Paz, Madrid, Spain; Interdisciplinary Department of Medicine (DIM), Rheumatology Unit, University of Bari, Bari, Italy; Rheumatology Department, Hospital Universitario de la Princesa, Instituto de Investigación Sanitaria (IIS) Princesa, Madrid; Unidad de Enfermedades Autoinmunes Sistémicas, Servicio de Medicina Interna, Universidad Autonoma de Barcelona, on behalf of the GEAS 
group, Barcelona, Spain; Department of Experimental and Clinical Medicine, and Department of Medical and Surgical Critical Care, Section of Dermatology, University of Florence, Florence; Unità Operativa Complessa (UOC) Reumatologia, Ospedale San Camillo-Forlanini, Rome; ACURA Centre for Rheumatic Diseases, Baden-Baden, Germany; Rheumatology Department, Città Della Salute e della Scienza, Torino; Division of Rheumatology, University and Institute for Research and Health Care (IRCCS) Policlinico S. Matteo Foundation, Pavia, Italy; Servicio de Reumatología, Hospital General Universitario Gregorio Marañón, Madrid, Spain; Dermatology Clinic, University Hospital of Trieste; Department of Pneumology and Respiratory Intermediate Care Unit, University Hospital of Cattinara, Trieste; Rheumatology Unit, Azienda Ospedaliero-Universitaria Ospedali Riuniti di Trieste, Trieste, Italy; Medizinische Klinik A, Klinikum der Stadt, Ludwigshafen, Germany, Research Laboratory and Academic Division of Clinical Rheumatology, Department of Internal Medicine, University of Genoa, San Martino Polyclinic Hospital IRCCS Genoa, Genoa, Italy; Interstitial and Rare Lung Disease Unit, Ruhrlandklinik University Hospital, University of Duisburg-Essen, Essen, Germany; UOC Reumatologia, Azienda Ospedaliero Universitaria S. Anna, University of Ferrara, Italy; Department for Rheumatology and Clinical Immunology, St. Josef Krankenhaus, University Clinic, Essen, Germany; Division of Rheumatology, Department of Clinical and Experimental Medicine, University of Pisa, Pisa; Department of Internal Medicine, Rheumatology and Clinical Immunology, University Hospital Johannes-Gutenberg, Mainz, Germany; University of Ghent, Ghent University Hospital, Ghent, Belgium.

M. Sebastiani, MD, Rheumatology Unit, Azienda Policlinico of Modena, University of Modena and Reggio Emilia; A. Manfredi, MD,

Rheumatology Unit, Azienda Policlinico of Modena, University of Modena and Reggio Emilia; G. Cassone, MD, Rheumatology Unit, Azienda Policlinico of Modena, University of Modena and Reggio Emilia; $K$. Triantafyllias, MD, ACURA Rheumatology Center; A. Schwarting, MD, Professor, ACURA Rheumatology Center; M.A. González-Gay, MD, Professor, Rheumatology Division, Hospital Universitario Marqués de Valdecilla, IDiVAL, University of Cantabria; N. Palmou-Fontana, MD, Rheumatology Division, Hospital Universitario Marqués de Valdecilla, IDiVAL, University of Cantabria; U. Drott, MD, Rheumatology Division, University Hospital of Frankfurt; C. Delbrück, MD, Rheumatology Division, University Hospital of Frankfurt; J. Rojas-Serrano, MD, Professor, Interstitial Lung Disease and Rheumatology Unit, Instituto Nacional de Enfermedades Respiratorias, Ismael Cosío Villegas, C. Bertolazzi, MD, Interstitial Lung Disease and Rheumatology Unit, Instituto Nacional de Enfermedades Respiratorias, Ismael Cosío Villegas; L. Nuño, MD, Servicio de Reumatología, Hospital Universitario La Paz; M. Giannini, MD, DIM, Rheumatology Unit, University of Bari; F. Iannone, MD, Professor, DIM, Rheumatology Unit, University of Bari; E.F. Vicente, MD, Rheumatology Department, Hospital Universitario de la Princesa, IIS Princesa; S. Castañeda, MD, Professor, Rheumatology Department, Hospital Universitario de la Princesa, IIS Princesa; A. Selva-O'Callaghan, MD, Professor, Unidad de Enfermedades Autoinmunes Sistémicas, Servicio de Medicina Interna, Universidad Autonoma de Barcelona, on behalf of the GEAS group; E. Trallero Araguas, MD, Unidad de Enfermedades Autoinmunes Sistémicas, Servicio de Medicina Interna, Universidad Autonoma de Barcelona, on behalf of the GEAS group; G. Emmi, MD, Department of Experimental and Clinical Medicine, University of Florence; A. Iuliano, MD, UOC Reumatologia, Ospedale San Camillo-Forlanini; J. Bauhammer, MD, ACURA Centre for Rheumatic Diseases; N. Miehle MD, ACURA Centre for Rheumatic Diseases; S. Parisi, MD, Rheumatology Department, Città Della Salute e della Scienza; L. Cavagna, MD, Division of Rheumatology, University and IRCCS Policlinico S. Matteo Foundation; V. Codullo, MD, Division of Rheumatology, University and IRCCS Policlinico S. Matteo Foundation; C. Montecucco, MD, Professor, Division of Rheumatology, University and IRCCS Policlinico S. Matteo Foundation; F.J. Lopez-Longo, MD, Servicio de Reumatología, Hospital General Universitario Gregorio Marañón; J. Martínez-Barrio, MD, Servicio de Reumatología, Hospital General Universitario Gregorio Marañón; J.C. Nieto-González, MD, Servicio de Reumatología, Hospital General Universitario Gregorio Marañón; S. Vichi, MD, Dermatology Clinic, University Hospital of Trieste; M. Confalonieri, MD, Department of Pneumology and Respiratory Intermediate Care Unit, University Hospital of Cattinara; P. Tomietto,
MD, Rheumatology Unit, Azienda Ospedaliero-Universitaria Ospedali Riuniti; R. Bergner, MD, Professor, Medizinische Klinik A, Klinikum der Stadt; A. Sulli, MD, Professor, Research Laboratory and Academic Division of Clinical Rheumatology, Department of Internal Medicine, University of Genoa, San Martino Polyclinic Hospital IRCCS Genoa; M. Cutolo, MD, Professor, Research Laboratory and Academic Division of Clinical Rheumatology, Department of Internal Medicine, University of Genoa, San Martino Polyclinic Hospital IRCCS Genoa; F. Bonella, MD, Interstitial and Rare Lung Disease Unit, Ruhrlandklinik University Hospital, University of Duisburg-Essen; F. Furini, MD, UOC Reumatologia, Azienda Ospedaliero Universitaria S. Anna, University of Ferrara; C.A. Scirè, MD, Associate Professor, UOC Reumatologia, Azienda Ospedaliero Universitaria S. Anna, University of Ferrara; A. Bortoluzzi, MD, UOC Reumatologia, Azienda Ospedaliero Universitaria S. Anna, University of Ferrara; C. Specker, MD, Professor, Department for Rheumatology and Clinical Immunology, St. Josef Krankenhaus, University Clinic; S. Barsotti, MD, Division of Rheumatology, Department of Clinical and Experimental Medicine, University of Pisa; R. Neri, MD, Division of Rheumatology, Department of Clinical and Experimental Medicine, University of Pisa; M. Mosca, MD, Professor, Division of Rheumatology, Department of Clinical and Experimental Medicine, University of Pisa; M. Caproni, MD, Department of Medical and Surgical Critical Care, Section of Dermatology, University of Florence; J. Weinmann-Menke, MD, Department of Internal Medicine, Rheumatology and Clinical Immunology, University Hospital Johannes-Gutenberg; A. Schwarting, MD, Professor, Department of Internal Medicine, Rheumatology and Clinical Immunology, University Hospital Johannes-Gutenberg; V. Smith, MD, Professor, University of Ghent, Ghent University Hospital. Dr. Sebastiani and Dr. Triantafyllias contributed equally to this article.

Address correspondence to Dr. K. Triantafyllias, ACURA Rheumatology Center,Bad Kreuznach, Germany.E-mail: ktriantafyllias@gmail.com Accepted for publication August 13, 2018.

Antisynthetase syndrome (AS) is a heterogeneous autoimmune disease mainly characterized by the classic triad of arthritis, myositis, and interstitial lung disease (ILD) $)^{1,2}$ and by the occurrence of antiaminoacyl tRNA-synthetase antibodies (anti-ARS). Raynaud phenomenon (RP), fever, and mechanic's hands are other relevant but less prevalent accompanying features ${ }^{3}$. The most frequent ARS antibody is the anti-Jo1, whereas other ARS specificities are less commonly detected (for example, anti-PL-7, -PL-12, -EJ, -OJ, -KS, - YRS, -Zo) $)^{1,4}$.

Nailfold videocapillaroscopy (NVC) is a safe, noninvasive diagnostic tool for the in vivo study of periungueal microcirculation, in particular for the examination of capillary density and morphology ${ }^{5}$. NVC is one of the most reliable diagnostic modalities to differentiate primary from secondary $\mathrm{RP}^{6}$, and it has been included among the 2013 classification criteria for systemic sclerosis $(\mathrm{SSc})^{7}$. Large-scale efforts are being made to standardize the evaluation of capillaroscopic morphology throughout rheumatic diseases through the European League Against Rheumatism study group on microcirculation $^{8,9}$.

Despite the increasing number of capillaroscopic studies in SSc reported over the last years, valid and systematic capillaroscopic evidence concerning AS are still lacking. The scarce NVC data come mainly from a few studies and case reports that assessed other conditions such as polymyositis (PM) and dermatomyositis (DM) $)^{10,11,12,13,14}$. These studies reported positivity for ARS antibodies only in small 
subgroups, making it difficult to extract detailed and reliable data concerning the role of these antibodies. Moreover, patients in these studies were not clearly characterized as having AS, possibly also because there are no well-established classification criteria for this rare disease ${ }^{15}$. AENEAS (the American and European NEtwork of Antisynthetase Syndrome) is an international collaborative group that aims to study AS thoroughly and better understand its clinical and pathophysiological features ${ }^{16,17}$.

By considering the relevance of accompanying findings in general and of RP in particular in $\mathrm{AS}^{18}$, this multicenter study (Nailfold Capillaroscopy in Antisynthetase Syndrome) aimed to describe the capillaroscopic features from a well-characterized cohort of patients, investigating possible correlations with clinical and serological features of the disease.

\section{MATERIALS AND METHODS}

Within the framework of the AENEAS collaborative group, we retrospectively evaluated all patients with AS who underwent NVC during their clinical history. In the study, we included patients with at least 2 antisynthetase antibody-positive tests, with 1 or more findings of the classic triad (arthritis, myositis, and ILD). Triad findings were identified as follows: arthritis occurrence was defined clinically by the referent physician, ILD by the occurrence of a restrictive pattern at pulmonary function test [forced vital capacity $(\mathrm{FVC}) \leq 80 \%$, forced expiratory volume (FEV) $1 / \mathrm{FVC} \geq 70 \%$, decreased/normal FEV1, and/or DLCO reduction $>20 \%$ ] and/or by the identification of alveolitis/fibrosis signs at high-resolution computed tomography of the lungs. Finally, muscle involvement was defined in case of any muscle enzyme elevation plus the presence of typical electromyography alterations and/or compatible muscle biopsy findings and/or suggestive muscle magnetic resonance findings. According to previous reports, the time of appearance of these 3 clinical manifestations (arthritis, ILD, myositis) was also recorded. Manifestations onset was considered concomitant if the delay in their appearance was $<3$ months. RP occurrence was objectively assessed by direct physician observation and/or by photographic documentation provided by patients. All patients were further investigated for mechanic's hand and smoking habit.

In keeping with the purpose of the study, patients meeting the classification criteria for $\mathrm{SSc}^{7}$ were excluded.

After Institutional Review Board approval (ethics committee of the Azienda Policlinico of Modena, Italy, no. 3857/C.E.), clinical characteristics of the disease, and laboratory and instrumental data were retrieved from patients' clinical records. Images ( 2 images per finger from the middle area of the nailfold) from every center were anonymized and then securely shared in a personalized storage folder for central reevaluation by an expert operator (MS) blinded to patients' clinical data. A second expert operator (AM) assessed blindly interoperator reproducibility of results in a sample of 50 random patients (interrater agreement $=0.85$ ). Capillaroscopic findings of patients with AS were compared with those of 75 controls, represented by patients with primary RP (female/male ratio 4.13/1, mean age 53.9 \pm 17.6 yrs), referring to a historical cohort of ANA-negative patients without any sign of connective tissue disease other than RP. A minimum of 2 years of followup was requested as an entry criterion in the control group. A comparison was performed also within the patients with AS, to detect autoantibody specificities (anti-Jo1 and non-anti-Jo1 antibodies positivity) and clinical pattern presentation.

Collection of NVC images. NVC was performed using different machines (Videocap software 3.0, DS Medica; Zeiss, Stemi 2000-C; Mediscope D1, Optilia; Dinocapture 2.0, Dino-Lite; Capiscope) equipped with a 100 or $200 \times$ optical probe, after patients had been in a comfortable temperature of $22-25^{\circ} \mathrm{C}$ for $20 \mathrm{~min}$. A drop of immersion oil was applied to the nailfold to maximize the translucency of the keratin layer, and the second through the fifth fingers of both hands were examined ${ }^{19}$. To increase the reproducibility among different videocapillaroscopies, all measurements were performed on $1 \mathrm{~mm}$.

According to previous definitions ${ }^{20,21,22}$, the following capillaroscopic variables were evaluated: giant capillaries (normally shaped homogeneously enlarged capillary with a limb diameter $\geq 50 \mu \mathrm{m}$ ); microhemorrhages (presence of one or more dark red masses characterized by hemosiderin deposits due to capillary injury or thrombosis); ramified capillaries (branching, bushy, interconnected capillaries, originating from a single capillary); the number of capillaries per linear $\mathrm{mm}$; the number of giant capillaries per linear mm; avascular area (intercapillaries distance $>500 \mu \mathrm{m}$ ). Because some NVC features are occasionally observed in only 1 or a few fingers without clinical significance, to better evaluate the severity of these capillaroscopic alterations, we also evaluated the percentages of images per patient with microhemorrhages, ramified capillaries, giant capillaries, and avascular areas. Moreover, an SSc pattern, defined as an alteration of the nailfold microvascular network characterized by enlarged and giant loops, microhemorrhages, capillary loss, ramified capillaries, and architectural disorganization $20,21,22,23,24$, was recorded as being present or absent. In the absence of validated criteria and of comparison studies, we choose to use the term "SSc-like" in the presence of an NVC pattern similar to the SSc pattern.

Statistical analysis. Data were expressed as mean \pm SD unless otherwise noted. Categorical variables were analyzed by chi-square test or Fisher's exact test as appropriate; Wilcoxon test was used to compare repeated measurements in paired groups, while differences between the means were determined using Mann-Whitney $\mathrm{U}$ test for unpaired samples. $\mathrm{P}$ values $\leq 0.05$ were considered statistically significant ${ }^{25}$.

\section{RESULTS}

A total of 2550 images (with a mean of $13.5 \pm 4.1$ images per subject) from 190 patients with AS were analyzed (females/males 3.63, mean age $49.7 \pm 12.8$ yrs, mean disease duration $51.2 \pm 71.4 \mathrm{mos})$.

RP was recorded in $43.2 \%$ of patients, arthritis in $76.5 \%$, myositis in $80.2 \%$, and ILD in $86.2 \%$. Anti-Jo1 antibodies were detected in 133 patients $(69.5 \%)$. On the other hand, 19 patients $(10 \%)$ were positive for anti-PL-7 antibodies, 22 (11.5\%) for anti-PL-12, $6(3 \%)$ for anti-OJ, and $10(6 \%)$ for anti-EJ.

The clinical, serological, and demographic features of the patients are reported in Table 1.

Comparison of patients with AS and control group. NVC abnormalities (at least 1 among giant capillaries, microhemorrhages, ramifications, reduction of the number/mm) were more frequently observed in patients with AS than in controls (62.1\% vs $29.3 \%$, respectively, p < 0.001).

Regarding NVC variables, giant capillaries and avascular areas were recorded only in the AS group (59 patients, $31.1 \%$ and 39 patients, $20.5 \%$, respectively, $\mathrm{p}<0.005)$. Ramified capillaries were more frequent in the AS group (94 patients, $49.5 \%$ vs 5 patients, $6.7 \%$, p < 0.005 ), and no differences were observed for microhemorrhages (44 patients, $23.2 \% \mathrm{vs}$ 18 patients, $24 \%, \mathrm{p}=$ not significant), although the degree of severity was higher in AS than in the control group $(\mathrm{p}=0.008)$. Microhemorrhages were also more pronounced in patients with $\mathrm{AS}$, and (reasonably) related to local trauma

Personal non-commercial use only. The Journal of Rheumatology Copyright @ 2019. All rights reserved. 
Table 1. Demographic, clinical, and serological features of 190 patients with antisynthetase syndrome.

\begin{tabular}{lc}
\hline Characteristics & Values \\
\hline Mean age, yrs, \pm SD & $49.7 \pm 12.8$ \\
Female/male ratio & 3.63 \\
Median disease duration, mos (IQR) & $53.7(82)$ \\
RP & 43.2 \\
Mechanic's hands & 41.1 \\
Arthritis & 76.5 \\
Myositis & 80.2 \\
Interstitial lung disease & 86.2 \\
Clinical triad* & \\
1 clinical manifestation & 12.3 \\
2 clinical manifestations & 39 \\
3 clinical manifestations & 48.7 \\
Antinuclear antibodies & 61.8 \\
Anti-SSA & 51.6 \\
Anti-Jo1 & 69.5 \\
Other antisynthetase antibodies & 32.3 \\
\hline
\end{tabular}

Data are percentages unless otherwise indicated. *Arthritis, myositis, interstitial lung disease. IQR: interquartile range; RP: Raynaud phenomenon.

in the control group. The number of capillaries was higher in the control group ( $\mathrm{p}<0.005$; details in Table 2 ).

If a pathologic pattern was by definition absent in the control group, an SSc-like pattern was found in the $35.3 \%$ of patients with AS (Table 2). Ramified capillaries were detected in almost $50 \%$ of patients with AS, while the concurrent presence of giant and bushy capillaries was recorded in $26.2 \%$ of patients with AS. Interestingly, giant and ramified capillaries were found in $73.8 \%$ of the patients with SSc-like pattern (Figure 1).

Association between NVC and demographic, clinical, and serological features of $A S$. In patients with $\mathrm{AS}$, the presence of at least $1 \mathrm{NVC}$ abnormality was associated with anti-Jo1 antibodies positivity $(\mathrm{p}=0.008)$ and presence of ILD $(\mathrm{p}=0.02)$. Interestingly, ramified capillaries were twice as frequent in patients with ILD as in patients without $(53.4 \%$ vs $27.6 \%$, respectively; $\mathrm{p}=0.01)$.

Table 2. Capillaroscopic features of patients with AS and controls.

\begin{tabular}{lccc}
\hline Variables & AS, $\mathrm{n}=190$ & Controls, $\mathrm{n}=75$ & $\mathrm{p}$ \\
\hline Frequency (\%) & & & \\
$\quad$ Microhemorrhages & 23.2 & 24 & $\mathrm{~ns}$ \\
Ramified capillaries & 49.5 & 6.7 & $<0.005$ \\
SSc-like pattern & 35.3 & 0 & $<0.005$ \\
No. per mm & & & \\
Capillaries & $8.49 \pm 2.27$ & $10.8 \pm 0.67$ & $<0.005$ \\
Lowest no. capillaries & $6.90 \pm 2.63$ & $9.27 \pm 0.86$ & $<0.005$ \\
$\quad$ No. giant capillaries & $0.18 \pm 0.39$ & 0 & $<0.005$ \\
Severity (no. images per patient with at least a finding/10) & \\
$\quad$ Microhemorrhages & $0.7 \pm 1.6$ & $0.2 \pm 0.3$ & 0.008 \\
Ramified capillaries & $2.3 \pm 3.1$ & $0.6 \pm 0.2$ & $<0.005$
\end{tabular}

SSc: systemic sclerosis; AS: antisynthetase syndrome.
Among the single NVC features, avascular areas, detected in $17.9 \%$ of patients, were significantly associated with autoimmunity markers (anti-SSA, anti-Jo1), myositis, and RP (Supplementary Data, available with the online version of this article).

An SSc-like pattern was associated with anti-Jo1 antibodies (44\% vs $21.5 \%$, in patients with anti-Jo1 antibodies and other antisynthetase specificities, respectively; $\mathrm{p}=0.002)$ and also with a longer disease duration (107.1 \pm 87.6 vs $84.6 \pm 85.9$ mos in patients with or without SSc-like pattern, respectively; $\mathrm{p}=0.004)$. Moreover, multivariate analysis showed a correlation between disease duration and SSc-like pattern (OR 2.73, 95\% CI 1.04-7.17; $\mathrm{p}=0.04$, Table $3)$. There was no correlation between an SSc-like pattern and the final number of clinical manifestations of the disease (ILD, arthritis, myositis, mechanic's hands, fever; all comparisons showing $\mathrm{p}>0.05)$, but SSc-like pattern was more commonly observed in patients with a later appearance of ILD (63 patients) regarding patients presenting ILD from disease onset (102 cases) or not presenting ILD (25 subjects; $\mathrm{p}=0.018$ ). A multivariate analysis confirmed the independent association between anti-Jo1 antibodies, disease duration, the late occurrence of ILD, and the presence of an SSc-like pattern (Table 3).

Interestingly, there was no significant correlation between the presence of SSc-like pattern and RP, and only $47 \%$ of patients with SSc-like pattern had RP.

\section{DISCUSSION}

To our knowledge, this is the first multicenter study examining NVC features systematically in a large population of patients with AS. Moreover, the study described for the first time the characteristics and the frequency of SSc-like pattern in patients with AS, and its correlation with the clinical features of the disease. The analysis of 190 patients with AS from participating centers allowed us to establish that NVC pattern of AS is abnormal regarding people with primary RP, thus confirming that vasculopathy is a typical manifestation of this rare condition. The more frequent NVC finding was ramified capillaries, while SSc-like pattern was observed in $35.3 \%$ of cases. A significant correlation was found between ILD and ramified capillaries, but not with SSc-like pattern.

Previously, only a few studies analyzed the relationship between anti-ARS and NVC features ${ }^{12,13}$. A small study concerning the NVC alterations in 24 patients with idiopathic inflammatory myopathies (IIM) reported a positivity of anti-Jo1 antibodies in 8 patients ${ }^{12}$. The only abnormality reported in this small cohort of anti-Jo1-positive patients was a reduction of the capillary density in comparison to other patients.

Positivity of anti-ARS was not associated with the presence of SSc-like pattern in a study on 13 patients with DM reported by Mugii, et al ${ }^{10}$. In contrast, our study showed

Personal non-commercial use only. The Journal of Rheumatology Copyright $\subset$ $\subset$ 2019. All rights reserved 


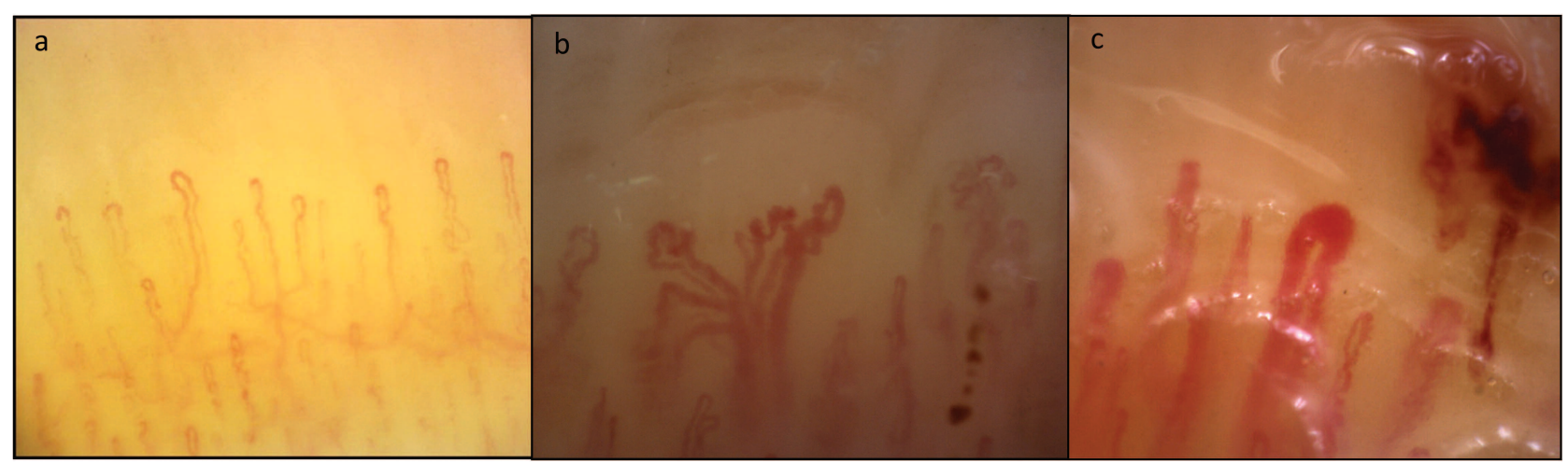

Figure 1. Different morphological patterns on nailfold videocapillaroscopy. A. Raynaud phenomenon (homogeneously distributed capillaries with normal architecture and morphology). B. and C. SSc-like pattern in antisynthetase syndrome: architectural disorganization, giant and ramified capillaries, microhemorrhages, reduction of the number of capillaries per linear mm. SSc: systemic sclerosis.

Table 3. Multivariate analysis. Association with SSc-like pattern.

\begin{tabular}{lcccc}
\hline Variables & OR & \multicolumn{2}{c}{$95 \%$ CI } & p \\
& & Lower & Higher & \\
\hline Disease duration $\geq 24$ mos & 2.731 & 1.041 & 7.167 & 0.041 \\
ILD $\geq$ second AS manifestation & 2.245 & 1.147 & 4.395 & 0.018 \\
Anti-Jo1 antibodies & 2.885 & 1.342 & 6.205 & $0.007 \mathrm{j} 1$ \\
\hline
\end{tabular}

SSc: systemic sclerosis; ILD: interstitial lung disease; AS: antisynthetase syndrome.

a significant association between anti-Jo1 antibodies and SSc-like pattern. Nevertheless, it is possible that the low number of patients in the subgroup analysis of Mugii, et al could have been the reason for the lack of a significant association $^{10}$

Selva-O'Callaghan, et al investigated 53 patients with $\mathrm{IIM}^{11}$ and reported a positivity for anti-ARS in $16(12$ positive for anti-Jo1 and 4 for other ARS). The comparison between patients with the 16 anti-ARS and 6 anti-Pm/Scl antibodies-positive patients did not show statistically significant differences in regard to NVC alterations.

Interestingly, in a study examining 27 patients with DM, an SSc-like pattern was recorded in 4/4 patients with anti-Jo1 antibodies ${ }^{13}$.

In our population, NVC abnormalities were detected in more than $60 \%$ of patients with AS, while an SSc-like pattern was observed in the $35.3 \%$ of cases.

NVC findings were differently associated with the main clinical features of AS. In this regard, NVC alterations were more frequently observed in patients with ILD compared to patients without $(\mathrm{p}=0.02)$, and myositis was associated to avascular areas $(\mathrm{p}=0.004)$, while no specific NVC features were detected in patients with arthritis. Interestingly, we observed no differences according to the final number of major clinical manifestations.

We previously observed a high prevalence of NVC alter- ations in patients with inflammatory muscle diseases, observing that NVC major alterations are quite exclusive of $\mathrm{DM}$, while $\mathrm{PM}$ was not different by primary $\mathrm{RP}^{20}$. Moreover, we observed differences between SSc pattern and DM pattern (observed in patients with DM). In DM, giant and ramified capillaries were seen at disease onset, while in patients with longer disease duration, DM pattern regressed and only brushing capillaries were observed ${ }^{23}$.

At present, we cannot establish whether patients with AS are characterized by true $\mathrm{SSc}$-specific patterns ${ }^{24}$ or DM pattern ${ }^{23}$ or by a specific AS pattern. For this reason, comparison studies of AS with SSc and DM are required to address this question, and the term SSc-like pattern appears to be more proper. In contrast to that observed in DM, we observed a direct correlation between disease duration and SSc-like pattern in patients with AS, suggesting a different phenotype of microangiopathy in AS and DM.

Remarkably, patients with SSc-like pattern at NVC seem to develop ILD late in the course of the disease. Prospective studies are needed to confirm this finding and to evaluate the possible value of NVC in the prediction of interstitial lung involvement. This point is not a secondary issue, because together with other clinical aspects such as the occurrence of de novo accompanying findings during followup ${ }^{18}$, NVC could result in an easily obtainable marker providing additional information on the degree of risk for the clinical spectrum progression in patients with AS.

Further, clinically relevant RP in AS is only associated with the presence of avascular areas, but not with SSc-like pattern. It is also worth noting that SSc-like pattern was found in $32.1 \%$ of patients without RP from our series and that only $47 \%$ of patients with SSc-like pattern had RP. This point highlights the practical value of performing NVC in patients with connective tissue disease, arthritis, or ILD, regardless of the presence of RP. In particular, all patients with rheumatoid arthritis or ILD with SSc-like pattern at NVC should be investigated for an underlying AS. Even if

Personal non-commercial use only. The Journal of Rheumatology Copyright @ 2019 . All rights reserved. 
inclusion of patients has not occurred at disease onset, findings of the investigation can also be of importance because clinical spectrum time course of this disease is highly variable, and clinical manifestations may occur even after years from disease onset ${ }^{15,16}$.

The major limitation of this study is its retrospective design. However, the high number of examined NVC and the large amount of clinical data made it possible to obtain clinically relevant and novel information. Nevertheless, longitudinal studies are needed and planned by our group to examine the temporal relationship between NVC findings and the clinical manifestations of AS in a prospective manner. An additional limitation could be that currently NVC is not routinely performed in patients with AS. Therefore, we could have a challenging bias in our population, with an overestimation of the prevalence of RP, the main indication of NVC.

AS is a complex entity, in which the different clinical manifestations are variably associated, determining a large spectrum of disease ${ }^{16}$. According to our results, microangiopathy should be defined as a hallmark of disease together with arthritis, myositis, and ILD, and in association with the most frequent serum biomarker of the disease (anti-Jo1), could allow identifying a more defined AS subtype.

Prospective studies are needed to confirm our data, in particular the potential predictive role of NVC for the subsequent occurrence of ILD. Further, our results suggest the need to better evaluate the role of microangiopathy in the pathogenesis of disease, the association with specific antibodies, and the possible association with disease clinical manifestations.

\section{ONLINE SUPPLEMENT}

Supplementary material accompanies the online version of this article.

\section{REFERENCES}

1. Chatterjee S, Prayson R, Farver C. Antisynthetase syndrome: Not just an inflammatory myopathy. Cleve Clin J Med 2013;80:655-66.

2. Dugar M, Cox S, Limaye V, Blumbergs P, Roberts-Thomson PJ. Clinical heterogeneity and prognostic features of South Australian patients with anti-synthetase autoantibodies. Intern Med J 2011;41:674-9.

3. Lega JC, Fabien N, Reynaud Q, Durieu I, Durupt S, Dutertre M, et al. The clinical phenotype associated with myositis-specific and associated autoantibodies: a meta-analysis revisiting the so-called antisynthetase syndrome. Autoimmun Rev 2014;13:883-91.

4. Hervier B, Devilliers H, Stanciu R, Meyer A, Uzunhan Y, Masseau A, et al. Hierarchical cluster and survival analyses of antisynthetase syndrome: phenotype and outcome are correlated with anti-tRNA synthetase antibody specificity. Autoimmun Rev 2012;12:210-7.

5. Cutolo M, Smith V. State of the art on nailfold capillaroscopy: a reliable diagnostic tool and putative biomarker in rheumatology? Rheumatology 2013;52:1933-40.

6. Cutolo M, Pizzorni C, Secchi ME, Sulli A. Capillaroscopy. Best Pract Res Clin Rheumatol 2008;22:1093-108.

7. van den Hoogen F, Khanna D, Fransen J, Johnson S. Classification criteria for systemic sclerosis: an ACR-EULAR collaborative initiative. Arthritis Rheum 2013;65:2737-47.
8. Smith V, Beeckman S, Herrick AL, Decuman S, Deschepper E, De Keyser F, et al. An EULAR study group pilot study on reliability of simple capillaroscopic definitions to describe capillary morphology in rheumatic diseases. Rheumatology 2016;55:883-90.

9. Cutolo M, Melsens K, Herrick AL, Foeldvari I, Deschepper E, De Keyser F, et al. Reliability of simple capillaroscopic definitions in describing capillary morphology in rheumatic diseases. Rheumatology 2018;57:757-9.

10. Mugii N, Hasegawa M, Matsushita T, Hamaguchi Y, Horie S, Yahata T, et al. Association between nail-fold capillary findings and disease activity in dermatomyositis. Rheumatology 2011;50:1091-8.

11. Selva-O'Callaghan A, Fonollosa-Pla V, Trallero-Araguás E, Martínez-Gómez X, Simeon-Aznar CP, Labrador-Horrillo M, et al. Nailfold capillary microscopy in adults with inflammatory myopathy. Semin Arthritis Rheum 2010;39:398-404.

12. Mercer LK, Moore TL, Chinoy H, Murray AK, Vail A, Cooper RG, et al. Quantitative nailfold video capillaroscopy in patients with idiopathic inflammatory myopathy. Rheumatology 2010; 49:1699-705.

13. Shenavandeh S, Zarei Nezhad M. Association of nailfold capillary changes with disease activity, clinical and laboratory findings in patients with dermatomyositis. Med J Islam Repub Iran 2015;29:586-90.

14. Riccieri V, Vasile M, Macri V, Sciarra I, Stefanantoni K, De Luca N, et al. Successful immunosuppressive treatment of dermatomyositis: a nailfold capillaroscopy survey. J Rheumatol 2010;37:443-5.

15. Cavagna L, Castañeda S, Sciré C, Gonzalez-Gay MA; AENEAS Collaborative Group Members. Antisynthetase syndrome or what else? Different perspectives indicate the need for new classification criteria. Ann Rheum Dis 2018;77:e50.

16. Cavagna L, Nuño L, Scirè CA, Govoni M, Longo FJ, Franceschini $\mathrm{F}$, et al. Clinical spectrum time course in anti Jo-1 positive antisynthetase syndrome: results from an international retrospective multicenter study. Medicine 2015;94:e1144.

17. González-Gay MA, Montecucco C, Selva-O'Callaghan A, Trallero-Araguas E, Molberg O, Andersson H, et al. Timing of onset affects arthritis presentation pattern in antisyntethase syndrome. Clin Exp Rheumatol 2018;36:44-9.

18. Bartoloni E, Gonzalez-Gay MA, Scirè C, Castaneda S, Gerli R, Lopez-Longo FJ, et al. Clinical follow-up predictors of disease pattern change in anti-Jo1 positive anti-synthetase syndrome: results from a multicenter, international and retrospective study. Autoimmun Rev 2017;16:253-7.

19. Etehad Tavakol M, Fatemi A, Karbalaie A, Emrani Z, Erlandsson BE. Nailfold capillaroscopy in rheumatic diseases: which parameters should be evaluated? Biomed Res Int 2015;2015:1-17.

20. Cutolo M, Sulli A, Smith V. How to perform and interpret capillaroscopy. Best Pract Res Clin Rheumatol 2013;27:237-48.

21. Manfredi A, Sebastiani M, Cassone G, Pipitone N, Giuggioli D, Colaci M, et al. Nailfold capillaroscopic changes in dermatomyositis and polymyositis. Clin Rheumatol 2015; 34:279-84.

22. Sulli A, Secchi ME, Pizzorni C, Cutolo M. Scoring the nailfold microvascular changes during the capillaroscopic analysis in systemic sclerosis patients. Ann Rheum Dis 2008;67:885-7.

23. Manfredi A, Sebastiani M, Campomori F, Pipitone N, Giuggioli D, Colaci M, et al. Nailfold videocapillaroscopy alterations in dermatomyositis and systemic sclerosis: toward identification of a specific pattern. J Rheumatol 2016;43:1575-80.

24. Cutolo M, Sulli A, Pizzorni C, Accardo A. Nailfold videocapillaroscopy assessment of microvascular damage in systemic sclerosis. J Rheumatol 2000;27:155-60.

25. Altman DG. Practical statistic for medical research. London: Chapman and Hall; 1991. 